\title{
Long Time Effect of Soil Solarization Integrated with Dazomet or Chicken Manure on Yield, Weeds and Root-Knot Nematodes in Tomato and Melon
}

\author{
Vincenzo Candido*1, Trifone D’Addabbo ${ }^{2}$, Martino Basile², Donato Castronuovo ${ }^{1}$, \\ Vito Miccolis ${ }^{1}$ \\ ${ }^{1}$ Dipartimento di Scienze dei Sistemi Colturali, Forestali e dell'Ambiente, Università della Basilicata \\ Viale dell'Ateneo Lucano 10, 85100 Potenza, Italy \\ ${ }^{2}$ CNR, Istituto per la Protezione delle Piante, Via G. Amendola 165/A, 70126 Bari, Italy
}

Received: 26 June 2008. Accepted: 20 October 2008.

\begin{abstract}
Effects of 5-week soil solarization, either alone or combined with dazomet or a chicken manure compost, on tomato and melon yield, root-knot nematode infestation and weeds were investigated along three crop cycles in a plastic greenhouse infested by Meloidogyne javanica in Southern Italy. Solarization treatment, either alone and combined with dazomet or organic amendment, always resulted in a significant increase of marketable crop yield, and its effect lasted up to two years from the treatment. Nematode population indices and number of root galls were always lower in solarized soil than in untreated control. Organic amendment alone suppressed soil nematode population only in the first two crop cycles, though less than solarization and with no significant reduction of gall formation. Dazomet alone resulted in a yield increase only in the first tomato crop, with no significant reduction of soil nematode density and root galls. Solarization treatment completely suppressed the emergence of all the annual and perennial weed species, though C. rotundus was controlled only immediately after the treatment. Suppressivity of SOL on annual weeds and the perennial $C$. dactylon was extended to the tomato and melon crop following the treatment, but persisted on $D$. sanguinalis and $P$. oleracea, also in the third crop. Combination of solarization with dazomet or chicken manure compost did not enhance the suppressive effect on weeds. Solarization confirmed to provide an effective suppression of root-knot nematodes and weeds in the greenhouse vegetable crop systems of warm climate regions, with no need to be combined with other control tools.
\end{abstract}

Key-words: greenhouse, solarization, dazomet, chicken manure, yield, weeds Meloidogyne javanica, melon, tomato.

\section{Introduction}

Soil solarization (SOL) is a cost-saving and environmentally safe technique for a nonchemical soil disinfestations (Katan and DeVay, 1991). Under appropriate climate conditions SOL can ensure an effective control of a wide range of pathogens, weeds and nematodes in different agricultural systems (Stapleton, 2000).

Root-knot nematodes (Meloidogyne spp.) are spread worldwide and cause a large part of the annual yield losses attributed to nematode damage (Trudgill and Blok, 2001). Environmental and health concerns imposed the with- drawal of most nematicides and soil fumigants available for the control of these phytoparasites and emphasized the need for alternative safer control strategies, including the use of SOL (Noling and Becker, 1994). Several field reports stated that root-knot nematode infestation may be consistently reduced for two consecutive years after the heat treatment (Stevens et al., 2003). However, the rapid recolonization of the soil following SOL may reduce its residual effectiveness, mainly in greenhouse conditions (Candido et al., 2008; Ioannou, 2000).

The effects of SOL against root-knot nematodes may be furtherly enhanced by the combi-

* Corresponding Author: Tel.: +39 0971 205371; Fax: +39 0971 205378. E-mail address: vincenzo.candido@unibas.it Authors equally shared the work. 
nation with other control tools, as low doses of fumigant nematicides, among which also dazomet (DAZ), or organic amendments.

Dazomet (DAZ) is a granular methyl isothiocyanate precursor known since the $60 \mathrm{~s}$ as a broad spectrum fumigant with limited environmental effects (Ruzo, 2006), active on soilborne pathogens, weeds and also root-knot nematodes (Giannakou et al., 2002; Gilreath et al., 2004).

Many composts from agroindustrial residues like chicken manure (CM) were found to be potentially suppressive on root-knot nematodes (Nico et al., 2004; Oka and Yermiyahu, 2002).

The combination of SOL with reduced doses of DAZ proved to enhance the suppressivity of thermal treatment either on root-knot nematodes (Yucel et al., 2007) and weeds (Benlioğlu et al., 2005). Integration of SOL with raw or composted $\mathrm{CM}$ was also proved to enhance the effect of thermal treatment on root-knot nematodes in various experiments, either in field (Gamliel and Stapleton, 1993) and in greenhouse (Oka et al., 2007; Kafikavalci, 2007).

However, most of investigations on the combined use of SOL and DAZ or CM were limited to a single crop cycle, with no or few information about the residual nematicidal and agronomical effects of these combined treatments on the following crop cycles. Therefore, a threeyear greenhouse experiment was undertaken in order to investigate the effect of combination of SOL with reduced rates of DAZ or CM soil amendments on yield parameters and infestation of the root-knot nematode $M$. javanica (Treub) Chitw. along three different crop cycles.

\section{Materials and methods}

The experiment was undertaken in a metal-plastic $(200-\mu \mathrm{m}$ thick low-density polyethylene transparent film) greenhouse located at Metaponto $\left(40^{\circ} 24^{\prime} \mathrm{N}, 16^{\circ} 28^{\prime} \mathrm{E}\right)$ in Southern Italy. The alkaline ( $\mathrm{pH} 8.4)$ sandy soil was poor at organic matter $\left(3.3 \mathrm{~g} \mathrm{~kg}^{-1}\right)$ and nitrogen $(0.5$ $\mathrm{g} / \mathrm{kg}$ ) and heavily infested (3.6 eggs and juveniles $\mathrm{cm}^{-3}$ soil) by $M$. javanica.

Soil was ploughed at $30 \mathrm{~cm}$ depth and uniformly rotavated after removing plant residues of the previous melon crop. Soil surface was then divided into three blocks, each subdivided into six $6 \times 4 \mathrm{~m}$ plots, spaced $1 \mathrm{~m}$ apart. Two
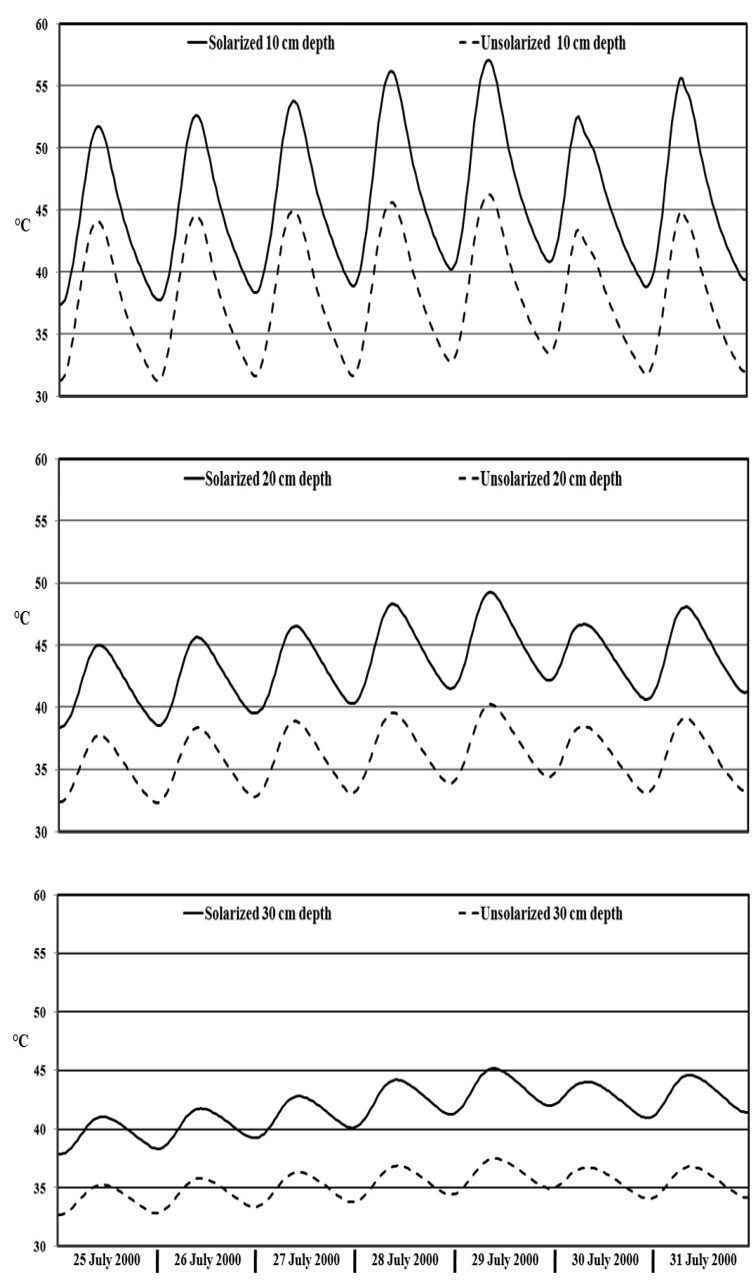

Figure 1. Temperature trend during the representative week (25 July - 31 July 2000) at $10 \mathrm{~cm}$ depth.

plots of each blocks were treated with $50 \mathrm{~g} \mathrm{~m}^{-2}$ granular DAZ, two others were amended with $40 \mathrm{t} \mathrm{ha}^{-1}$ of a CM-based compost (CM, grape and olive pomace and grapestone meal) and the last two were left untreated. DAZ and CM compost were incorporated into the soil by rotavation and then plots were irrigated to field capacity at $30 \mathrm{~cm}$ depth through a drip irrigation system with dripper lines $0.5 \mathrm{~m}$ apart and emitters $\left(3 \mathrm{~L} \mathrm{~h}^{-1}\right.$ water flow rate) spaced $0.20 \mathrm{~m}$ from each other. A 34-day SOL was then applied to a half of each block by covering with a $35 \mu \mathrm{m}$ thick ethylene-vinyl acetate (EVA) solarizing film on 17 July 2000, two weeks after CM amendment and two days after DAZ treatment, respectively. Therefore a total of 6 treatments, SOL alone or combined with DAZ or CM com- 
post, DAZ or CM compost alone and non-treated soil, were arranged in a randomized complete block design with three replicates.

During SOL, soil temperatures at 10, 20 and $30 \mathrm{~cm}$ depth were monitored at 60-min intervals by $\mathrm{T}-100$ probes and a CR- 10X data-logger (Campbell Scientific, Inc., USA) either in solarized and non-solarized soil.

At the end of SOL period, tomato (Lycopersicon esculentum Mill.) cv Naxos $\mathrm{F}_{1}$ (having an indefinite habit) one month-old seedlings were transplanted in rows $1 \mathrm{~m}$ apart (3.3 plants $\mathrm{m}^{-2}$ ) on 24 August 2000 and tomato fruits were harvested from 27 November 2000 to 26 January 2001. On 23 March 2001, melon (Cucumis melo L. var. reticulatus Naud. cv Drake $\mathrm{F}_{1}$ ) was directly sown in rows $2 \mathrm{~m}$ apart $\left(0.5\right.$ plants $\left.\mathrm{m}^{-2}\right)$, after a $15 \mathrm{~cm}$ soil rotavation and the complete removal of tomato crop residues. Fruits were harvested from 22 June to 6 July 2001. In the following spring 'cherry' tomato cv Tomito $\mathrm{F}_{1}$ (a dwarf plant genotype) was transplanted in rows $1 \mathrm{~m}$ apart, 3.3 plants $\mathrm{m}^{-2}$, on 28 March 2002, whereas the fruit bunches were harvested from 17 to 27 June. During the three crop cycles soil was mulched with a black LDPE plastic film and plants received fertilizer application and irrigation according to the vegetable cultural practices of the area.

The effect of different treatments on plant growth was evaluated by recording the flowering date of each flower cluster in the first tomato crop and the plant dry weight and leaf area in the following melon crop on 5 plants from each subplot.

Number and weight of marketable fruits, average fruit weight and fruit soluble solids ( ${ }^{\circ}$ Brix) and dry matter content were assessed on the 10 tomato or 6 melon central plants in each subplot at the end of each crop cycle. Moreover, nematode infestation (root gall index) was estimated on the roots of the same plants, according to a $0-5$ scale in which $0=$ no galls, $1=1$ to 2 galls, $2=3$ to 10 galls, $3=11$ to 30 galls, $4=31$ to 100 galls and $5=>100$ galls (Taylor and Sasser, 1987). At the same time, a composite soil sample of 40 cores was collected from $0-30 \mathrm{~cm}$ soil layer with a soil probe, $1.5 \mathrm{~cm}$ diameter and $30 \mathrm{~cm}$ long, in the center square meter of each plot. Eggs and juveniles were extracted from $500 \mathrm{~cm}^{-3}$ aliquots by Coolen (1979)'s method and counted. Moreover, after solarization treatment and at the end of each crop cycle the weeds from a $2-\mathrm{m}^{2}$ sampling area in the center of each plot and from the nonmulched soil between the rows were counted and classified and their dry weight was determined. Weed biomass was completely removed from the soil after each observation.

Daily temperature trend at 10,20 and $30 \mathrm{~cm}$ depth, either in solarized and non solarized soil, was graphically described with reference to a representative solarization week (25 July - 31 July 2000), whereas the cumulative number of hours of temperature permanence above $40{ }^{\circ} \mathrm{C}$ was calculated for the whole solarization period. Nematode and weed data were statistically analyzed after $\operatorname{Ln}(\mathrm{x}+1)$ transformation for homogenization of error variances. All data were statistically analyzed by ANOVA, and means separated by Fisher's Least Significant Difference Test at $\alpha \leq 0.05$.

\section{Results}

\subsection{Effects on soil temperatures}

During the representative solarization week, temperatures in the first $10 \mathrm{~cm}$ solarized soil were always higher than in nonheated control and persisted constantly above $37^{\circ} \mathrm{C}$ either during the day and over the night (Fig. 1). In solarized soil, peak temperatures at $20 \mathrm{~cm}$ depth were lower than in the upper $10 \mathrm{~cm}$, though ranging just below $50{ }^{\circ} \mathrm{C}$, whereas the lowest thermal values never decreased under $38{ }^{\circ} \mathrm{C}$. Temperatures in solarized soil were still higher than $37^{\circ} \mathrm{C}$ also at $30 \mathrm{~cm}$ depth.

Considering the whole solarization period, temperatures in solarized soil persisted in the range $40-45^{\circ} \mathrm{C}$ for 301,404 and 595 hours at 10 , 20 and $30 \mathrm{~cm}$ depth, respectively, whereas only 173,7 and 0 hours, respectively at 10, 20 and 30 $\mathrm{cm}$ depth, were totalled in the nonsolarized soil (Tab. 1). A lower cumulative number of hour was recorded in the $46-50{ }^{\circ} \mathrm{C}$ interval, as a permanence of 176,276 and $74 \mathrm{hrs}$ in solarized soil and of 48, 0 and $0 \mathrm{hrs}$ in the nonsolarized soil was reached, respectively at 10, 20 and $30 \mathrm{~cm}$ depth. Only the upper $10 \mathrm{~cm}$ layer of solarized soil totalled 63 and 43 hours of permanence in the $51-55{ }^{\circ} \mathrm{C}$ and $>56{ }^{\circ} \mathrm{C}$ temperature ranges, respectively. 
Table 1. Cumulative number of hours of temperature permanence above $40{ }^{\circ} \mathrm{C}$ at 10,20 and $30 \mathrm{~cm}$ depth in solarized and nonsolarized soil.

\begin{tabular}{|c|c|c|c|c|c|c|c|c|c|c|c|c|}
\hline \multirow[t]{3}{*}{ Treatment } & \multicolumn{12}{|c|}{ Temperature ranges } \\
\hline & \multicolumn{3}{|c|}{$40-45^{\circ} \mathrm{C}$} & \multicolumn{3}{|c|}{$46-50^{\circ} \mathrm{C}$} & \multicolumn{3}{|c|}{$51-55^{\circ} \mathrm{C}$} & \multicolumn{3}{|c|}{$\geq 56{ }^{\circ} \mathrm{C}$} \\
\hline & $10 \mathrm{~cm}$ & $20 \mathrm{~cm}$ & $30 \mathrm{~cm}$ & $10 \mathrm{~cm}$ & $20 \mathrm{~cm}$ & $30 \mathrm{~cm}$ & $10 \mathrm{~cm}$ & $20 \mathrm{~cm}$ & $30 \mathrm{~cm}$ & $10 \mathrm{~cm}$ & $20 \mathrm{~cm}$ & $30 \mathrm{~cm}$ \\
\hline Solarize & 301 & 404 & 595 & 176 & 276 & 74 & 163 & 0 & 0 & 43 & 0 & 0 \\
\hline Nonsolarized & 173 & 7 & 0 & 48 & 0 & 0 & 0 & 0 & 0 & 0 & 0 & 0 \\
\hline
\end{tabular}

\subsection{Effects on crop yield}

In the first tomato cycle SOL treatment resulted in a faster tomato plant growth and in a significantly earlier formation and blooming of flower clusters, particularly evident on the $3^{\text {rd }}$ and $4^{\text {th }}$ clusters, 12 and 16 days earlier than the unsolarized plots, respectively, whereas flowering of other clusters occurred 5-7 days before (Fig. 2). Blooming of plants in DAZ-treated soil was from 2 to 7 days earlier than control, whereas an intermediate behaviour was found in plants from CM-treated soil. SOL significantly increased marketable yield (+182\% as a mean) and number of fruits per plant (+ $107 \%)$, compared to non-solarized treatments (Tab. 2). Moreover, a significantly higher weight and a lower content of dry matter and soluble solids were also found in the fruits from plants in solarized soil.

SOL positively affected also plant growth parameters in the following melon crop, as plant dry weight and leaf area at thinning (25 days after sowing) were significantly higher in solarized plots than in the untreated control or in DAZ or CM-treated soil (Fig. 3). Marketable fruits from SOL -treated soil were also significantly more $(+350 \%)$, heavier $(+461 \%)$ and with a

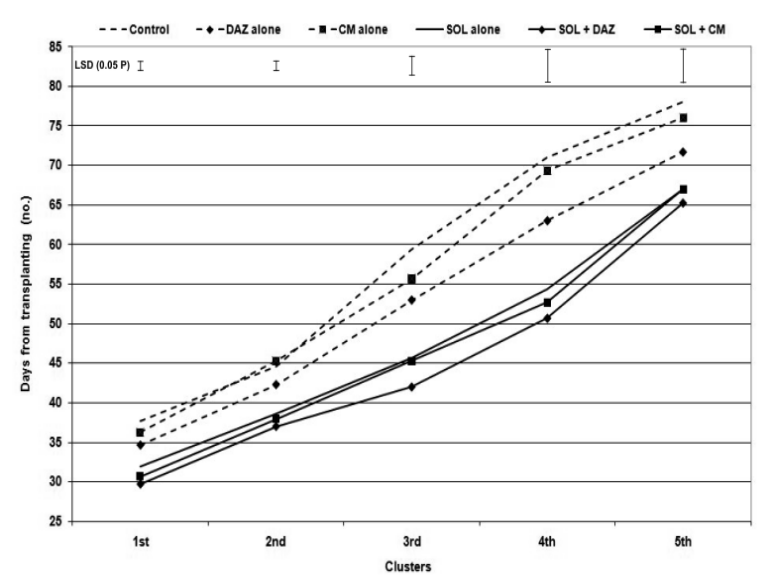

Figure 2. Effect of soil solarization (SOL), Dazomet (DAZ) or composted chicken manure (CM) either alone or in combination, on blooming time of different flower clusters in the tomato cv Naxos crop.

higher dry matter and soluble solids content than in the control (Tab. 3). The effects of SOL was extended also to the yield of the third crop cycle, as tomato yield of solarized plots was increased by $76 \%$ compared to nonsolarized ones, due to a higher number (+ $49 \%)$ and weight $(+34 \%)$ of fruits, whereas no statistical difference was found for dry matter and soluble solids content (Tab. 4).

Table 2. Effect of soil solarization (SOL), either alone or combined with dazomet (DAZ) or composted chicken manure $(\mathrm{CM})$ on tomato cv Naxos yield parameters and infestation of the root-knot nematode Meloidogyne javanica.

\begin{tabular}{|c|c|c|c|c|c|c|c|}
\hline \multirow[t]{3}{*}{ Treatment } & \multicolumn{5}{|c|}{ Yield parameters } & \multirow{2}{*}{\multicolumn{2}{|c|}{$\begin{array}{c}\text { M. javanica infestation } \\
\text { parameters }\end{array}$}} \\
\hline & \multicolumn{2}{|c|}{ Marketable yield } & \multicolumn{3}{|c|}{ Fruit quality } & & \\
\hline & $\left(\mathrm{T} \mathrm{ha}{ }^{-1}\right)$ & $\begin{array}{c}\text { Number of } \\
\text { fruits per plant }\end{array}$ & $\begin{array}{l}\text { Weight } \\
\text { (g) }\end{array}$ & $\begin{array}{c}\text { Dry matter } \\
(\%)\end{array}$ & $\begin{array}{l}\text { Soluble solids } \\
\left({ }^{\circ} \text { Brix }\right)\end{array}$ & $\begin{array}{l}\text { Root gall } \\
\text { index }\end{array}$ & $\begin{array}{c}\text { Eggs and } \mathrm{J}_{2} \\
\mathrm{ml}^{-1} \text { soil }\end{array}$ \\
\hline Control & $16.6 \mathrm{~d}$ & $6.1 \mathrm{~d}$ & $80.5 \mathrm{~b}$ & $7.2 \mathrm{a}$ & $5.5 \mathrm{a}$ & $4.9 \mathrm{a}$ & $6.1 \mathrm{a}$ \\
\hline DAZ alone & $26.0 \mathrm{c}$ & $8.7 \mathrm{c}$ & $88.7 \mathrm{~b}$ & $6.1 \mathrm{~b}$ & $4.9 \mathrm{~b}$ & $4.9 \mathrm{a}$ & $5.4 \mathrm{a}$ \\
\hline $\mathrm{CM}$ alone & $16.9 \mathrm{~d}$ & $5.9 \mathrm{~d}$ & $85.2 \mathrm{~b}$ & $6.8 \mathrm{a}$ & $4.9 \mathrm{~b}$ & $4.3 \mathrm{a}$ & $2.91 \mathrm{~b}$ \\
\hline SOL alone & $49.9 \mathrm{~b}$ & $13.0 \mathrm{~b}$ & $115.6 \mathrm{a}$ & $5.8 \mathrm{bc}$ & $4.3 \mathrm{c}$ & $0.1 \mathrm{~b}$ & $0.6 \mathrm{c}$ \\
\hline $\mathrm{SOL}+\mathrm{DAZ}$ & $63.1 \mathrm{a}$ & $16.0 \mathrm{a}$ & $118.3 \mathrm{a}$ & $5.4 \mathrm{c}$ & $4.1 \mathrm{c}$ & $0.1 \mathrm{~b}$ & $0.5 \mathrm{c}$ \\
\hline $\mathrm{SOL}+\mathrm{CM}$ & $54.7 \mathrm{~b}$ & $13.8 \mathrm{~b}$ & $118.4 \mathrm{a}$ & $5.7 \mathrm{bc}$ & $4.1 \mathrm{c}$ & $0.0 \mathrm{~b}$ & $0.5 \mathrm{c}$ \\
\hline
\end{tabular}

Data followed by the same letters on the same column are not significantly $(\mathrm{P}=0.05)$ different according to Least Significant Difference's Test. 
Table 3. Effect of soil solarization (SOL), either alone or combined with dazomet (DAZ) or composted chicken manure (CM) on melon cv Drake yield parameters and infestation of the root-knot nematode Meloidogyne javanica.

\begin{tabular}{|c|c|c|c|c|c|c|c|}
\hline \multirow[t]{3}{*}{ Treatment } & \multicolumn{5}{|c|}{ Yield parameters } & \multirow{2}{*}{\multicolumn{2}{|c|}{$\begin{array}{c}\text { M. javanica infestation } \\
\text { parameters }\end{array}$}} \\
\hline & \multicolumn{2}{|c|}{ Marketable yield } & \multicolumn{3}{|c|}{ Fruit quality } & & \\
\hline & $\left(\mathrm{T} \mathrm{ha}{ }^{-1}\right)$ & $\begin{array}{l}\text { Number of } \\
\text { fruits per plant }\end{array}$ & $\begin{array}{l}\text { Weight } \\
\text { (g) }\end{array}$ & $\begin{array}{c}\text { Dry matter } \\
(\%)\end{array}$ & $\begin{array}{l}\text { Soluble solids } \\
\left.\text { ( }{ }^{\circ} \text { Brix }\right)\end{array}$ & $\begin{array}{l}\text { Root gall } \\
\text { index }\end{array}$ & $\begin{array}{l}\text { Eggs and } \mathrm{J}_{2} \\
\mathrm{ml}^{-1} \text { soil }\end{array}$ \\
\hline Control & $10.8 \mathrm{~b}$ & $1.5 \mathrm{~b}$ & $1.9 \mathrm{a}$ & $5.4 \mathrm{c}$ & $8.8 \mathrm{c}$ & $5.0 \mathrm{a}$ & $5.7 \mathrm{a}$ \\
\hline DAZ alone & $6.2 \mathrm{~b}$ & $0.7 \mathrm{~b}$ & $2.2 \mathrm{a}$ & $6.1 \mathrm{bc}$ & $10.1 \mathrm{bc}$ & $5.0 \mathrm{a}$ & $5.5 \mathrm{a}$ \\
\hline $\mathrm{CM}$ alone & $9.1 \mathrm{~b}$ & $1.2 \mathrm{~b}$ & $1.9 \mathrm{a}$ & $5.6 \mathrm{bc}$ & $9.7 \mathrm{bc}$ & $5.0 \mathrm{a}$ & $3.1 \mathrm{~b}$ \\
\hline SOL alone & $50.2 \mathrm{a}$ & $5.4 \mathrm{a}$ & $1.9 \mathrm{a}$ & $7.9 \mathrm{a}$ & $12.6 \mathrm{a}$ & $0.9 \mathrm{~b}$ & $0.4 \mathrm{c}$ \\
\hline $\mathrm{SOL}+\mathrm{DAZ}$ & $47.4 \mathrm{a}$ & $5.3 \mathrm{a}$ & $2.0 \mathrm{a}$ & $7.0 \mathrm{ab}$ & $11.2 \mathrm{ab}$ & $1.0 \mathrm{~b}$ & $0.4 \mathrm{c}$ \\
\hline $\mathrm{SOL}+\mathrm{CM}$ & $48.7 \mathrm{a}$ & $5.6 \mathrm{a}$ & $1.8 \mathrm{a}$ & $8.2 \mathrm{a}$ & $12.2 \mathrm{a}$ & $0.8 \mathrm{~b}$ & $0.3 \mathrm{c}$ \\
\hline
\end{tabular}

Data followed by the same letters on the same column are not significantly $(\mathrm{P}=0.05)$ different according to Least Significant Difference's Test.

Table 4. Effect of soil solarization (SOL), either alone or combined with dazomet (DAZ) or composted chicken manure (CM) on tomato cv Tomito yield parameters and infestation of the root-knot nematode Meloidogyne javanica.

\begin{tabular}{|c|c|c|c|c|c|c|c|}
\hline \multirow[t]{3}{*}{ Treatment } & \multicolumn{5}{|c|}{ Yield parameters } & \multirow{2}{*}{\multicolumn{2}{|c|}{$\begin{array}{c}\text { M. javanica infestation } \\
\text { parameters }\end{array}$}} \\
\hline & \multicolumn{2}{|c|}{ Marketable yield } & \multicolumn{3}{|c|}{ Fruit quality } & & \\
\hline & $\left(\mathrm{T} \mathrm{ha}{ }^{-1}\right)$ & $\begin{array}{l}\text { Number of } \\
\text { fruits per plant }\end{array}$ & $\begin{array}{l}\text { Weight } \\
\text { (g) }\end{array}$ & $\begin{array}{l}\text { Dry matter } \\
(\%)\end{array}$ & $\begin{array}{l}\text { Soluble solids } \\
\quad\left({ }^{\circ} \text { Brix }\right)\end{array}$ & $\begin{array}{l}\text { Root gall } \\
\text { index }\end{array}$ & $\begin{array}{l}\text { Eggs and } \mathrm{J}_{2} \\
\mathrm{ml}^{-1} \text { soil }\end{array}$ \\
\hline Control & $20.0 \mathrm{~b}$ & $69.0 \mathrm{~b}$ & $16.0 \mathrm{~b}$ & $7.4 \mathrm{a}$ & $6.8 \mathrm{a}$ & $4.8 \mathrm{a}$ & $4.1 \mathrm{a}$ \\
\hline DAZ alone & $22.8 \mathrm{~b}$ & $72.0 \mathrm{~b}$ & $14.9 \mathrm{~b}$ & $7.5 \mathrm{a}$ & $7.0 \mathrm{a}$ & $4.2 \mathrm{a}$ & $4.0 \mathrm{a}$ \\
\hline $\mathrm{CM}$ alone & $21.4 \mathrm{~b}$ & $75.3 \mathrm{~b}$ & $14.4 \mathrm{~b}$ & $7.0 \mathrm{a}$ & $6.5 \mathrm{a}$ & $4.5 \mathrm{a}$ & $4.2 \mathrm{a}$ \\
\hline SOL alone & $37.0 \mathrm{a}$ & $112.0 \mathrm{a}$ & $19.0 \mathrm{a}$ & $7.1 \mathrm{a}$ & $6.7 \mathrm{a}$ & $0.8 \mathrm{~b}$ & $1.8 \mathrm{~b}$ \\
\hline $\mathrm{SOL}+\mathrm{DAZ}$ & $38.4 \mathrm{a}$ & $108.0 \mathrm{a}$ & $18.4 \mathrm{a}$ & $7.3 \mathrm{a}$ & $6.7 \mathrm{a}$ & $0.5 \mathrm{~b}$ & $1.9 \mathrm{~b}$ \\
\hline $\mathrm{SOL}+\mathrm{CM}$ & $37.7 \mathrm{a}$ & $103.1 \mathrm{a}$ & $18.7 \mathrm{a}$ & $7.8 \mathrm{a}$ & $7.0 \mathrm{a}$ & $1.1 \mathrm{~b}$ & $1.7 \mathrm{~b}$ \\
\hline
\end{tabular}

Data followed by the same letters on the same column are not significantly $(\mathrm{P}=0.05)$ different according to Least Significant Difference's Test.

Compared to the nontreated soil, application of DAZ alone resulted in a significantly higher number of fruits and marketable yield and in

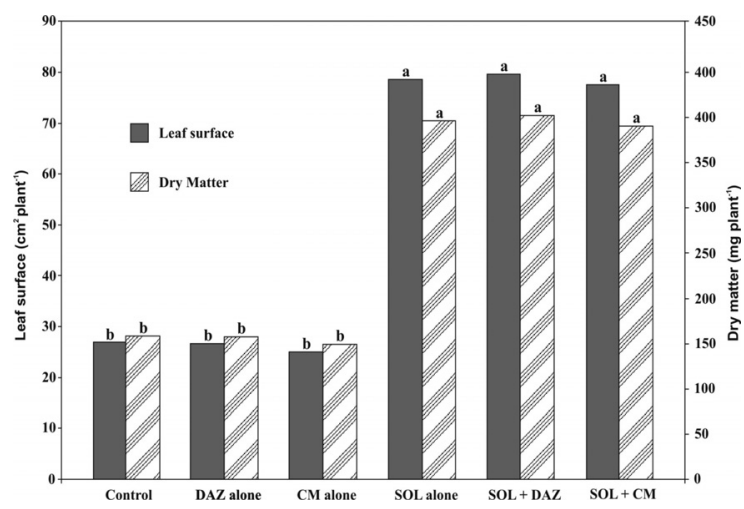

Figure 3. Effect of soil solarization (SOL), Dazomet (DAZ) or composted chicken manure (CM) either alone or in combination, on plant dry weight and leaf surface at thinning in the melon cv Drake crop.

Data of each character followed by the same letters are not significantly $(\mathrm{P}=0.05)$ different according to Least Significant Difference's Test. lower dry matter and soluble solid content only in the first crop cycle, whereas no further effect was found in the following melon and "cherry' tomato. However, yield and fruit quality parameters of DAZ-treated soil were always significantly worse than in solarized plots. Application of $\mathrm{CM}$ alone significantly affected only the fruit soluble solid content in the first tomato crop, but no other effect on crop yield derived from this treatment.

No significant improvement of the effect of SOL alone on yield parameters was achieved by its combination with DAZ or CM compost.

\subsection{Effects on root-knot nematodes}

In the first tomato cycle and in following melon crop the heat treatment significantly reduced the soil population of $M$. javanica and the formation of root galls compared to non-treated control and DAZ or CM alone (Tabb. 2-3). No significant increase of this nematicidal effect derived from the combination of SOL with $\mathrm{CM}$ or 
Table 5. Effect of soil solarization (SOL), either alone or combined with Dazomet (DAZ) or composted chicken manure (CM) on weed emergence at the end of soil solarization.

\begin{tabular}{|c|c|c|c|c|c|c|c|c|}
\hline \multirow[t]{3}{*}{ Treatment } & \multicolumn{4}{|c|}{ Perennial species } & \multicolumn{4}{|c|}{ Annual species } \\
\hline & \multicolumn{2}{|c|}{$\begin{array}{c}\text { Cyperus rotundus } \\
\text { L. }\end{array}$} & \multicolumn{2}{|c|}{$\begin{array}{l}\text { Cynodon dactylon } \\
\text { (L.) Pers. }\end{array}$} & \multicolumn{2}{|c|}{$\begin{array}{l}\text { Digitaria sanguinalis } \\
\text { (L.) P.B. }\end{array}$} & \multicolumn{2}{|c|}{$\begin{array}{l}\text { Portulaca oleracea } \\
\text { L. }\end{array}$} \\
\hline & $\begin{array}{c}\text { Number } \\
\left(\mathrm{n} \mathrm{m}^{-2}\right)\end{array}$ & $\begin{array}{l}\text { Dry weight } \\
\left(\mathrm{g} \mathrm{m}^{-2}\right)\end{array}$ & $\begin{array}{c}\text { Number } \\
\left(\mathrm{n} \mathrm{m}^{-2}\right)\end{array}$ & $\begin{array}{l}\text { Dry weight } \\
\left(\mathrm{g} \mathrm{m}^{-2}\right)\end{array}$ & $\begin{array}{c}\text { Number } \\
\left(\mathrm{n} \mathrm{m}^{-2}\right)\end{array}$ & $\begin{array}{l}\text { Dry weight } \\
\left(\mathrm{g} \mathrm{m}^{-2}\right)\end{array}$ & $\begin{array}{c}\text { Number } \\
\left(\mathrm{n} \mathrm{m}^{-2}\right)\end{array}$ & $\begin{array}{c}\text { Dry weight } \\
\left(\mathrm{g} \mathrm{m}^{-2}\right)\end{array}$ \\
\hline Control & $7.3 \mathrm{a}$ & $13.5 \mathrm{a}$ & $1.0 \mathrm{a}$ & $2.7 \mathrm{a}$ & $26.0 \mathrm{a}$ & $17.0 \mathrm{a}$ & $22.3 \mathrm{a}$ & $57.7 \mathrm{a}$ \\
\hline DAZ alone & $7.3 \mathrm{a}$ & $13.7 \mathrm{a}$ & $1.3 \mathrm{a}$ & $3.7 \mathrm{a}$ & $3.0 \mathrm{~b}$ & $1.0 \mathrm{~b}$ & $1.7 \mathrm{~b}$ & $6.0 \mathrm{~b}$ \\
\hline $\mathrm{CM}$ alone & $8.0 \mathrm{a}$ & $15.0 \mathrm{a}$ & $1.0 \mathrm{a}$ & $2.7 \mathrm{a}$ & $27.3 \mathrm{a}$ & $20.8 \mathrm{a}$ & $3.0 \mathrm{~b}$ & $10.6 \mathrm{~b}$ \\
\hline SOL alone & $0 \mathrm{~b}$ & $0 \mathrm{~b}$ & $0 \mathrm{~b}$ & $0 \mathrm{~b}$ & $0 \mathrm{~b}$ & $0 \mathrm{~b}$ & $0 \mathrm{~b}$ & $0 \mathrm{c}$ \\
\hline $\mathrm{SOL}+\mathrm{DAZ}$ & $0 \mathrm{~b}$ & $0 \mathrm{~b}$ & $0 \mathrm{~b}$ & $0 \mathrm{~b}$ & $0 \mathrm{~b}$ & $0 \mathrm{~b}$ & $0 \mathrm{~b}$ & $0 \mathrm{c}$ \\
\hline $\mathrm{SOL}+\mathrm{CM}$ & $0 \mathrm{~b}$ & $0 \mathrm{~b}$ & $0 \mathrm{~b}$ & $0 \mathrm{~b}$ & $0 \mathrm{~b}$ & $0 \mathrm{~b}$ & $0 \mathrm{~b}$ & $0 \mathrm{c}$ \\
\hline
\end{tabular}

Data followed by the same letters on the same column are not significantly $(\mathrm{P}=0.05)$ different according to Least Significant Difference's Test.

DAZ. Soil amendment with CM did not result in any significant effect on root galling but significantly suppressed nematode population compared to control and DAZ alone. Nematode infestation was not significantly affected by the only application of DAZ. At the end of the second tomato crop nematode population density and number of root galls resulted significantly reduced only in the soil previously solarized, whereas DAZ or CM-treated plots were not different from non-treated control (Tab. 4).

\subsection{Effects on weeds}

At the end of solarization period, all the heattreated plots, either with SOL alone or combined with DAZ or CM, were completely free of weeds, as high soil temperatures stopped germination or shooting of weed seeds or propagules, included the heat-resistant species (Tab. 5). Two poliennal, Cyperus rotundus L. and Cyn- odon dactylon (L.) Pers., and two annual, Digitaria sanguinalis (L.) Scop. and Portulaca oleracea L., weed species were found in nonsolarized soil. Compared to nontreated control, DAZ significantly reduced the number and the dry weight of both annual species, whereas CM treatment was suppressive only on $P$. oleracea. Presence of poliennal weeds was not significantly influenced by both DAZ and CM treatments.

Weed infestation was low in the autumn-winter tomato crop following SOL treatment. Heating soil, alone or combined with DAZ or CM, completely eliminated either annual $D$. sanguinalis and P. oleracea and perennial C. dactylon, whereas no effect was found on C. rotundus (Tab. 6). Emergence of both annual species was significantly lower in DAZ-treated soil than in CM-treated or untreated plots.

Melon crop in spring 2001 was prevalently

Table 6. Effect of soil solarization (SOL), either alone or combined with Dazomet (DAZ) or composted chicken manure (CM) on weed emergence in 'Naxos' tomato crop.

\begin{tabular}{|c|c|c|c|c|c|c|c|c|}
\hline \multirow[t]{3}{*}{ Treatment } & \multicolumn{4}{|c|}{ Perennial species } & \multicolumn{4}{|c|}{ Annual species } \\
\hline & \multicolumn{2}{|c|}{$\begin{array}{c}\text { Cyperus rotundus } \\
\text { L. }\end{array}$} & \multicolumn{2}{|c|}{$\begin{array}{l}\text { Cynodon dactylon } \\
\text { (L.) Pers. }\end{array}$} & \multicolumn{2}{|c|}{$\begin{array}{l}\text { Digitaria sanguinalis } \\
\text { (L.) P.B. }\end{array}$} & \multicolumn{2}{|c|}{$\begin{array}{l}\text { Portulaca oleracea } \\
\text { L. }\end{array}$} \\
\hline & $\begin{array}{c}\text { Number } \\
\left(\mathrm{n} \mathrm{m}^{-2}\right)\end{array}$ & $\begin{array}{l}\text { Dry weight } \\
\left(\mathrm{g} \mathrm{m}^{-2}\right)\end{array}$ & $\begin{array}{c}\text { Number } \\
\left(\mathrm{n} \mathrm{m}^{-2}\right)\end{array}$ & $\begin{array}{c}\text { Dry weight } \\
\left(\mathrm{g} \mathrm{m}^{-2}\right)\end{array}$ & $\begin{array}{c}\text { Number } \\
\left(\mathrm{n} \mathrm{m}^{-2}\right)\end{array}$ & $\begin{array}{l}\text { Dry weight } \\
\left(\mathrm{g} \mathrm{m}^{-2}\right)\end{array}$ & $\begin{array}{c}\text { Number } \\
\left(\mathrm{n} \mathrm{m}^{-2}\right)\end{array}$ & $\begin{array}{l}\text { Dry weight } \\
\left(\mathrm{g} \mathrm{m}^{-2}\right)\end{array}$ \\
\hline Control & $1.2 \mathrm{a}$ & $2.3 \mathrm{a}$ & $2.7 \mathrm{a}$ & $3.5 \mathrm{a}$ & $3.4 \mathrm{a}$ & $4.3 \mathrm{a}$ & $3.9 \mathrm{a}$ & $9.6 \mathrm{a}$ \\
\hline DAZ alone & $1.3 \mathrm{a}$ & $2.5 \mathrm{a}$ & $2.5 \mathrm{a}$ & $3.7 \mathrm{a}$ & $0.6 \mathrm{~b}$ & $0.9 \mathrm{~b}$ & $0.9 \mathrm{~b}$ & $2.2 \mathrm{~b}$ \\
\hline $\mathrm{CM}$ alone & $1.4 \mathrm{a}$ & $2.6 \mathrm{a}$ & $2.3 \mathrm{a}$ & $3.4 \mathrm{a}$ & $3.5 \mathrm{a}$ & $4.0 \mathrm{a}$ & $3.1 \mathrm{a}$ & $8.7 \mathrm{a}$ \\
\hline SOL alone & $1.0 \mathrm{a}$ & $2.2 \mathrm{a}$ & $0.0 \mathrm{~b}$ & $0.0 \mathrm{~b}$ & $0.0 \mathrm{~b}$ & $0.0 \mathrm{~b}$ & $0.0 \mathrm{~b}$ & $0.0 \mathrm{~b}$ \\
\hline $\mathrm{SOL}+\mathrm{DAZ}$ & $1.5 \mathrm{a}$ & $2.9 \mathrm{a}$ & $0.0 \mathrm{~b}$ & $0.0 \mathrm{~b}$ & $0.0 \mathrm{~b}$ & $0.0 \mathrm{~b}$ & $0.0 \mathrm{~b}$ & $0.0 \mathrm{~b}$ \\
\hline $\mathrm{SOL}+\mathrm{CM}$ & $1.2 \mathrm{a}$ & $2.4 \mathrm{a}$ & $0.0 \mathrm{~b}$ & $0.0 \mathrm{~b}$ & $0.0 \mathrm{~b}$ & $0.0 \mathrm{~b}$ & $0.0 \mathrm{~b}$ & $0.0 \mathrm{~b}$ \\
\hline
\end{tabular}

Data followed by the same letters on the same column are not significantly $(\mathrm{P}=0.05)$ different according to Least Significant Difference's Test. 
Table 7. Effect of soil solarization (SOL), either alone or combined with Dazomet (DAZ) or composted chicken manure $(\mathrm{CM})$ on weed emergence in melon crop.

\begin{tabular}{|c|c|c|c|c|c|c|c|c|c|c|c|c|}
\hline \multirow[t]{3}{*}{ Treatment } & \multicolumn{8}{|c|}{ Perennial species } & \multicolumn{4}{|c|}{ Annual species } \\
\hline & \multicolumn{2}{|c|}{$\begin{array}{c}\text { Cyperus } \\
\text { rotundus } \\
\mathrm{L} .\end{array}$} & \multicolumn{2}{|c|}{$\begin{array}{l}\text { Cynodon } \\
\text { dactylon } \\
\text { (L.) Pers. }\end{array}$} & \multicolumn{2}{|c|}{$\begin{array}{l}\text { Digitaria } \\
\text { sanguinalis } \\
\text { (L.) P.B. }\end{array}$} & \multicolumn{2}{|c|}{$\begin{array}{c}\text { Portulaca } \\
\text { oleracea } \\
\text { L. }\end{array}$} & \multicolumn{2}{|c|}{$\begin{array}{c}\text { Solanum } \\
\text { nigrum } \\
\text { L. }\end{array}$} & \multicolumn{2}{|c|}{$\begin{array}{c}\text { Melilotus } \\
\text { sulcatus } \\
\text { Desf. }\end{array}$} \\
\hline & $\begin{array}{c}\text { Number } \\
\left(\mathrm{n} \mathrm{m}^{-2}\right)\end{array}$ & $\begin{array}{c}\text { Dry } \\
\text { weight } \\
\left(\mathrm{g} \mathrm{m}^{-2}\right)\end{array}$ & $\begin{array}{c}\text { Number } \\
\left(\mathrm{n} \mathrm{m}^{-2}\right)\end{array}$ & $\begin{array}{c}\text { Dry } \\
\text { weight } \\
\left(\mathrm{g} \mathrm{m}^{-2}\right)\end{array}$ & $\begin{array}{c}\text { Number } \\
\left(\mathrm{n} \mathrm{m}^{-2}\right)\end{array}$ & $\begin{array}{c}\text { Dry } \\
\text { weight } \\
\left(\mathrm{g} \mathrm{m}^{-2}\right)\end{array}$ & $\begin{array}{c}\text { Number } \\
\left(\mathrm{n} \mathrm{m}^{-2}\right)\end{array}$ & $\begin{array}{c}\text { Dry } \\
\text { weight } \\
\left(\mathrm{g} \mathrm{m}^{-2}\right)\end{array}$ & $\begin{array}{l}\text { Number } \\
\left(\mathrm{n} \mathrm{m}^{-2}\right)\end{array}$ & $\begin{array}{c}\text { Dry } \\
\text { weight } \\
\left(\mathrm{g} \mathrm{m}^{-2}\right)\end{array}$ & $\begin{array}{c}\text { Number } \\
\left(\mathrm{n} \mathrm{m}^{-2}\right)\end{array}$ & $\begin{array}{c}\text { Dry } \\
\text { weight } \\
\left(\mathrm{g} \mathrm{m}^{-2}\right)\end{array}$ \\
\hline Control & $8.2 \mathrm{a}$ & 18.9 a & $2.7 \mathrm{a}$ & $17.0 \mathrm{a}$ & $148.7 \mathrm{a}$ & $372.5 \mathrm{a}$ & $13.3 \mathrm{a}$ & $54.4 \mathrm{a}$ & $13.3 \mathrm{a}$ & $126.7 \mathrm{a}$ & $0.0 \mathrm{c}$ & $0.0 \mathrm{c}$ \\
\hline DAZ alone & $8.0 \mathrm{a}$ & $19.5 \mathrm{a}$ & $2.4 \mathrm{a}$ & $15.8 \mathrm{a}$ & $10.6 \mathrm{~b}$ & $39.7 \mathrm{~b}$ & $2.3 \mathrm{~b}$ & $10.9 \mathrm{~b}$ & $0.0 \mathrm{~b}$ & $0.0 \mathrm{~b}$ & $0.0 \mathrm{c}$ & $0.0 \mathrm{c}$ \\
\hline $\mathrm{CM}$ alone & $7.7 \mathrm{a}$ & $18.1 \mathrm{a}$ & $2.4 \mathrm{a}$ & $14.8 \mathrm{a}$ & 106.6 a & $273.6 \mathrm{a}$ & $10.7 \mathrm{a}$ & $47.5 \mathrm{a}$ & $8.0 \mathrm{a}$ & $73.3 \mathrm{a}$ & $0.0 \mathrm{c}$ & $0.0 \mathrm{c}$ \\
\hline SOL alone & $7.5 \mathrm{a}$ & $19.1 \mathrm{a}$ & $0.0 \mathrm{~b}$ & $0.0 \mathrm{~b}$ & $0.0 \mathrm{~b}$ & $0.0 \mathrm{~b}$ & $0.0 \mathrm{~b}$ & $0.0 \mathrm{~b}$ & $0.0 \mathrm{~b}$ & $0.0 \mathrm{~b}$ & $10.7 \mathrm{a}$ & $84.4 \mathrm{a}$ \\
\hline $\mathrm{SOL}+\mathrm{DAZ}$ & $5.3 \mathrm{a}$ & $12.5 \mathrm{a}$ & $0.0 \mathrm{~b}$ & $0.0 \mathrm{~b}$ & $0.0 \mathrm{~b}$ & $0.0 \mathrm{~b}$ & $0.0 \mathrm{~b}$ & $0.0 \mathrm{~b}$ & $0.0 \mathrm{~b}$ & $0.0 \mathrm{~b}$ & $6.7 \mathrm{~b}$ & $56.7 \mathrm{~b}$ \\
\hline $\mathrm{SOL}+\mathrm{CM}$ & $8.0 \mathrm{a}$ & $20.1 \mathrm{a}$ & $0.0 \mathrm{~b}$ & $0.0 \mathrm{~b}$ & $0.0 \mathrm{~b}$ & $0.0 \mathrm{~b}$ & $0.0 \mathrm{~b}$ & $0.0 \mathrm{~b}$ & $0.0 \mathrm{~b}$ & $0.0 \mathrm{~b}$ & $8.0 \mathrm{~b}$ & $65.1 \mathrm{~b}$ \\
\hline
\end{tabular}

Data followed by the same letters on the same column are not significantly $(\mathrm{P}=0.05)$ different according to Least Significant Difference's Test.

infested by four annual species, mostly D. sanguinalis (Tab. 7). All the SOL treatments completely suppressed annuals D. sanguinalis, $P$. oleracea and Solanum nigrum L., and the poliennal C. dactylon. Density of $C$. rotundus was statistically not different in all the six treatments whereas SOL alone and, at a less extent, SOL + DAZ or CM, significantly increased the density of the annual species Melilotus sulcatus Desf. compared to nontreated soil. Significant reductions of plant density and dry weight were caused by DAZ alone on D. sanguinalis, $P$. oleracea and S. nigrum and by CM alone on M. sulcatus.

Tomato crop of spring 2002 was infested by many weeds, particularly annual species (Tab. 8). Number and dry biomass of $D$. sanguinalis and $P$. oleracea were still significantly lower in SOLtreated than in untreated soil, whereas no statistical difference was found for the emergence of annual $S$. nigrum and $M$. sulcatus and the perennial $C$. rotundus and $C$. dactylon. DAZ and CM, either alone and combined with SOL did not exert any further reduction of weed emergence.

\section{Discussion}

\subsection{Soil temperatures}

SOL treatment succeeded in raising soil temperatures at $0-30 \mathrm{~cm}$ depth above the lethal thresholds estimated for most soil pathogens and weeds. Ruiz et al. (2003) found $\mathrm{LD}_{95}$ values of 813,281 and $32.4 \mathrm{~min}$ at 39,42 , and $46{ }^{\circ} \mathrm{C}$,

Table 8. Effect of soil solarization (SOL), either alone or combined with Dazomet (DAZ) or composted chicken manure (CM) on weed emergence in spring tomato crop (cv Tomito).

\begin{tabular}{|c|c|c|c|c|c|c|c|c|c|c|c|c|}
\hline \multirow[t]{3}{*}{ Treatment } & \multicolumn{8}{|c|}{ Perennial species } & \multicolumn{4}{|c|}{ Annual species } \\
\hline & \multicolumn{2}{|c|}{$\begin{array}{l}\text { Cyperus } \\
\text { rotundus } \\
\mathrm{L} .\end{array}$} & \multicolumn{2}{|c|}{$\begin{array}{l}\text { Cynodon } \\
\text { dactylon } \\
\text { (L.) Pers. }\end{array}$} & \multicolumn{2}{|c|}{$\begin{array}{c}\text { Digitaria } \\
\text { sanguinalis } \\
\text { (L.) P.B. }\end{array}$} & \multicolumn{2}{|c|}{$\begin{array}{c}\text { Portulaca } \\
\text { oleracea } \\
\text { L. }\end{array}$} & \multicolumn{2}{|c|}{$\begin{array}{l}\text { Solanum } \\
\text { nigrum } \\
\text { L. }\end{array}$} & \multicolumn{2}{|c|}{$\begin{array}{l}\text { Melilotus } \\
\text { sulcatus } \\
\text { Desf. }\end{array}$} \\
\hline & $\begin{array}{c}\text { Number } \\
\left(\mathrm{n} \mathrm{m}^{-2}\right)\end{array}$ & $\begin{array}{c}\text { Dry } \\
\text { weight } \\
\left(\mathrm{g} \mathrm{m}^{-2}\right)\end{array}$ & $\begin{array}{c}\text { Number } \\
\left(\mathrm{n} \mathrm{m}^{-2}\right)\end{array}$ & $\begin{array}{c}\text { Dry } \\
\text { weight } \\
\left(\mathrm{g} \mathrm{m}^{-2}\right)\end{array}$ & $\begin{array}{c}\text { Number } \\
\left(\mathrm{n} \mathrm{m}^{-2}\right)\end{array}$ & $\begin{array}{c}\text { Dry } \\
\text { weight } \\
\left(\mathrm{g} \mathrm{m}^{-2}\right)\end{array}$ & $\begin{array}{l}\text { Number } \\
\left(\mathrm{n} \mathrm{m}^{-2}\right)\end{array}$ & $\begin{array}{c}\text { Dry } \\
\text { weight } \\
\left(\mathrm{g} \mathrm{m}^{-2}\right)\end{array}$ & $\begin{array}{l}\text { Number } \\
\left(\mathrm{n} \mathrm{m}^{-2}\right)\end{array}$ & $\begin{array}{c}\text { Dry } \\
\text { weight } \\
\left(\mathrm{g} \mathrm{m}^{-2}\right)\end{array}$ & $\begin{array}{l}\text { Number } \\
\left(\mathrm{n} \mathrm{m}^{-2}\right)\end{array}$ & $\begin{array}{c}\text { Dry } \\
\text { weight } \\
\left(\mathrm{g} \mathrm{m}^{-2}\right)\end{array}$ \\
\hline Control & $5.1 \mathrm{a}$ & $11.9 \mathrm{a}$ & $1.5 \mathrm{a}$ & $3.4 \mathrm{a}$ & $28.1 \mathrm{a}$ & $76.2 \mathrm{a}$ & $7.3 \mathrm{a}$ & $22.1 \mathrm{a}$ & $3.3 \mathrm{a}$ & $19.2 \mathrm{a}$ & $1.0 \mathrm{a}$ & $8.0 \mathrm{a}$ \\
\hline DAZ alone & $5.0 \mathrm{a}$ & $12.5 \mathrm{a}$ & $1.5 \mathrm{a}$ & $3.8 \mathrm{a}$ & $30.1 \mathrm{a}$ & $81.1 \mathrm{a}$ & $6.9 \mathrm{a}$ & $25.1 \mathrm{~b}$ & $2.0 \mathrm{a}$ & $16.0 \mathrm{a}$ & $0.7 \mathrm{a}$ & $6.2 \mathrm{a}$ \\
\hline $\mathrm{CM}$ alone & $4.6 \mathrm{a}$ & $12.1 \mathrm{a}$ & $1.4 \mathrm{a}$ & $4.3 \mathrm{a}$ & $32.0 \mathrm{a}$ & $73.1 \mathrm{a}$ & $5.9 \mathrm{a}$ & $19.2 \mathrm{a}$ & $1.7 \mathrm{a}$ & $15.0 \mathrm{a}$ & $0.8 \mathrm{a}$ & $7.6 \mathrm{a}$ \\
\hline SOL alone & $4.7 \mathrm{a}$ & $11.1 \mathrm{a}$ & $1.3 \mathrm{a}$ & $4.0 \mathrm{a}$ & $5.9 \mathrm{~b}$ & $11.0 \mathrm{~b}$ & $0.7 \mathrm{~b}$ & $2.3 \mathrm{~b}$ & $2.1 \mathrm{a}$ & $20.0 \mathrm{a}$ & $1.3 \mathrm{a}$ & $10.4 \mathrm{a}$ \\
\hline $\mathrm{SOL}+\mathrm{DAZ}$ & $3.9 \mathrm{a}$ & $12.3 \mathrm{a}$ & $1.6 \mathrm{a}$ & $3.9 \mathrm{a}$ & $4.3 \mathrm{~b}$ & $9.6 \mathrm{~b}$ & $0.3 \mathrm{~b}$ & $1.6 \mathrm{~b}$ & $3.0 \mathrm{a}$ & $27.0 \mathrm{a}$ & $0.9 \mathrm{a}$ & $9.1 \mathrm{a}$ \\
\hline $\mathrm{SOL}+\mathrm{CM}$ & $5.1 \mathrm{a}$ & $10.1 \mathrm{a}$ & $1.2 \mathrm{a}$ & $4.3 \mathrm{a}$ & $3.9 \mathrm{~b}$ & $10.6 \mathrm{~b}$ & $0.8 \mathrm{~b}$ & $2.6 \mathrm{~b}$ & $1.9 \mathrm{a}$ & $17.2 \mathrm{a}$ & $0.6 \mathrm{a}$ & $7.1 \mathrm{a}$ \\
\hline
\end{tabular}

Data followed by the same letters on the same column are not significantly $(\mathrm{P}=0.05)$ different according to Least Significant Difference's Test. 
respectively, for the root-knot nematode $M$. incognita Kofoid et White Chitw. after the application of constant temperature-time dosages to infested soil. $\mathrm{LD}_{50}$ of seeds of 8 common weed species was found at 50 to $66{ }^{\circ} \mathrm{C}$ at 12 hours of exposure (Egley, 1990), whereas Rubin and Benjamin (1984) reported that a 30-min exposure at a 30 to $90{ }^{\circ} \mathrm{C}$ temperature range decreased the viability of $C$. rotundus L. tuber in an inverse linear manner. Number of hours of lethal temperatures depends primarily on climate conditions, but can be related also to other factors, including soil structure, color, organic matter content and seedbed preparation (Grinstein and Hetzoni, 1991).

\subsection{Crop yields}

Faster tomato and melon growth in solarized plots, as testified by the earlier flowering and the heavier plants, respectively, may be related to the increased nitrogen availability in heattreated soil already reported by other authors (Chen et al., 2000; Grunzweig et al., 1999). Short-term availability of soluble forms of nitrogen, and particularly $\mathrm{NH}_{4}^{+}$and $\mathrm{NO}_{3}^{-}$fractions, was usually found increased after solarization, due to the higher decomposition rates of organic matter and the mineralization of microbial biomass killed by heat (Chen and Katan, 1980; Grunzweig et al., 1999).

Positive effects of soil solarization on crop yield emerging in our experiment have been largely documented since many years (Davis, 1991; Gamiel and Katan, 1991; Stapleton and Devay, 1984) and resulted particularly evident under greenhouse conditions, where crop yield and quality was found to last for more than two crop cycles (Candido et al., 2008). Larger fruit size and consequent higher water content can explain the lower soluble solids content of tomato fruits from solarized soil, whereas the higher soluble solids content of melon fruits was due to the presence of larger plants bearing a higher number of smaller size fruits consequent to the growth stimulation effect of solarization. Irregular ripening following early nematode attack and plant collapse may explain the low quality of melon fruits from nonsolarized plots. Stapleton and DeVay (1984) suggested that the beneficial effect of thermal treatment on crop yield can be related not only to the suppression of nematodes and weeds, but also to the release of nutrients induced by high soil temperatures. Residual effect of SOL on crop yield and fruit quality was extended to the two following crop cycles, but the long time effect of solar heating was already known from previous studies either in field (Katan et al., 1983) and particularly in greenhouse (Candido et al., 2008; Ioannou, 2000).

Yield response of DAZ was positive, though limited to the first crop cycle. The high mineralizing power of DAZ and the consequent increased nitrogen availability in DAZ-treated soil may account for the plant growth stimulation and yield increase effects of this fumigant (Scopa and Dumontet, 2007). Absence of a yield effect of CM amendment, either alone or combined with SOL, in all the three crop cycles is in contrast with previous literature, always reporting an increased crop yield as following CM soil amendments, mostly due to the CM effect on the major soil nutrients (Reddy et al., 2008) and to the suppression of soil pathogens and/or the modification of soil microbial community structure (Carrera et al., 2007; Hoitink and Boehm, 1999).

\subsection{Root-knot nematodes}

The SOL treatment suppressed nematode population and root gall formation along all the three crop cycles, confirming the excellent control of root-knot nematodes by SOL in greenhouse known since many years (Cartia, 1998). In more recent greenhouse experiments, the solarizing treatment drastically reduced plant infestation and root galling by $M$. javanica on tomato (Candido et al., 2008), as well as almost eradicated root-knot nematode population (Ostrec and Rubisic, 2003). In contrast to our results, the residual effectiveness of SOL was generally found to be limited by the greater soil depths inhabited by phytopathogenic nematodes and their rapid migration to upper soil layers after the treatment, as resulting in a quick recolonization of solarized soil (Stapleton and Heald, 1991).

DAZ treatment alone failed to control $M$. javanica infestation in our experiment, but previous greenhouse studies generally reported an effective control of root-knot nematode by preplant treatments with DAZ (Giannakou et al., 2002; Nagesh and Parvatha Reddy, 2005). Effectiveness of DAZ was found strictly related to 
optimal soil temperature, moisture and texture conditions (Fritsch and Huber, 1995): therefore failure of any of these conditions may have compromised treatment effect. Moreover DAZ, as mostly a fungicide, may have suppressed soil population of nematode antagonistic or parasitic fungi, allowing a quick soil recolonization by phytonematodes. Adversely, the population of $M$. javanica was consistently reduced by $\mathrm{CM}$ amendment alone along two crop cycles, confirming CM suppressivity on root-knot nematodes reported in a number of previous studies (D'Addabbo et al., 2003; Kaplan et al., 1992). Nematotoxicity of CM was generally related to the release of ammoniacal compounds (Tenuta and Lazarovits, 2002; Ben-Yephet et al., 2005), though also the effect on trophic diversity in soil nematode communities and the increase of nematode-antagonistic microbial population should be evaluated (Koenning and Barker, 2004; Riegel et al., 1996).

In this experiment no further improvement of nematicidal activity of SOL derived from the combination with a DAZ or CM treatment, but in previous greenhouse trials integration of SOL with low doses of DAZ decreased population densities of root-knot nematodes (Yucel et al., 2007). Combining SOL with CM was also previously reported to be potentially synergic and effective in reducing root-knot nematodes. Gamliel and Stapleton (1993) controlled M. incognita efficiently and increased lettuce yield by adding a CM compost to soil before SOL, and in more recent greenhouse trials SOL and $\mathrm{CM}$ were more effective when combined than alone in reducing the soil population of rootknot nematodes and gall formation on tomato and pepper plants (Kafikavalci, 2007; Oka et al., 2007).

Moreover, in a field experiments over two cropping seasons a 7 -week SOL combined with $10 \mathrm{~T} \mathrm{ha}^{-1} \mathrm{CM}$, provided also an effective control of most annual weed species (Benlioğlu et al., 2005). Gamliel et al. (1999) reported that the high temperatures raised by SOL increase the generation of toxic compounds by $\mathrm{CM}$, enhancing toxic activity against soil borne pathogens, nematodes and weeds. Oka et al. (2007) suggested that the plastic film would entrap higher concentrations of ammonia in the soil for longer periods, thus enabling a more effective diffusion in the soil. Finally, the combination of
SOL with a CM amendment may also improve the chemical fertility and impact positively the microbiological parameters of soil, as organic matter exerts a protective role on soil microbial biomass and enzymatic activities against the detrimental effect of heating (Clark et al., 2007; Scopa and Dumontet, 2007).

\subsection{Weeds}

SOL treatment completely suppressed the emergence of all the annual and perennial weed species, though $C$. rotundus was controlled only immediately after the treatment and recovered fastly in the following crop cycles. SOL effects on weed population were hypothesized to be due to different mechanisms, as changes in cell metabolism and ultrastructure (Singla et al., 1997), microbial parasitism on seeds weakened by sublethal temperatures, seed dormancy interruption by raising temperatures, foliar scorching of weeds under the plastic mulch (Egley, 1990).

A satisfactory SOL control of annual weeds was reported also in the previous studies. Stapleton et al. (2005) found that solar heating reduced by nearly $100 \%$ a wide range of annual weeds, including Melilotus spp and Digitaria spp., and Candido et al. (2008) reported that the emergence of many annual species, among which also D. sanguinalis and S. nigrum, was almost completely suppressed by greenhouse SOL. In a weed classification based on heat sensitivity, $P$. oleracea, was reported as undefined behaviour species (Restuccia et al., 1994), though Dahlquist et al. (2007) found a $39{ }^{\circ} \mathrm{C}$ temperature sublethal to seeds of $P$. oleracea, and infestation of this species was drastically reduced by a field SOL treatment (Patricio et al., 2006). Most perennial weeds were generally indicated as more difficult to control than annual species, maybe due to the occurrence of propagules at soil depths not exposed to lethal temperature (Rubin and Benjamin, 1984). Failure of SOL for the control of Cyperus spp. was reported by many studies (Candido et al., 2008; Stapleton et al., 2005), though a number of reports documented also an effective control of Cyperus spp and other perennial weeds by combining the SOL treatment with low rates of herbicides (Gilreath et al., 2005), or extending the length of SOL period (Chase et al., 1998). In the above cited classification of Restuccia et al. 
(1994), C. dactylon was ranked as an undefined behaviour species, though Rubin and Benjamin (1984) had reported the heat-sensitivity of this perennial weed.

Suppressivity of SOL on annual weeds and the perennial $C$. dactylon extended throughout the tomato and melon crop following the treatment, but the effects on $D$. sanguinalis and $P$. oleracea persisted, though at a lower size, also in the third crop. The residual effect of SOL treatment on weeds was reported by literature as much more pronounced than on nematodes and most fungal pathogens, as Candido et al. (2008) reported a consistent reduction or a total suppression of annual species and some perennial species after greenhouse SOL throughout the two following years and also later for $C$. dactylon. In other trials, soil was weedfree for at least three years after SOL in an olive orchard (Lopez-Escudero and BlancoLopez, 2001), and residual effects of SOL were observed on Cyperus spp. during four consecutive cropping seasons in a tomato-cucumber rotation (Gilreath et al., 2005). Moreover, Bell and Elmore (1983) found that persistence of weed control may be prolonged by the absence of soil disturbances after SOL treatment.

Combination of SOL with DAZ or CM did not enhance the suppressive effect on weeds. Integration with low rates of fumigants was previously found to improve SOL suppressiveness on weeds (Peachey et al., 2001), but the sequence of treatments was shown to play an important role in the final result (Eshel et al., 2000). Moreover, field and greenhouse trials demonstrated also a synergism of SOL with CM for weed control (Benlioglu et al., 2005; Haidar and Sidahmed, 2000).

DAZ treatment exerted a satisfactory control of annual weeds in the first two crop cycles, but was not effective on poliennal species, confirming the mixed results emerged from previous experiments (Landschoot et al., 2004; Locascio et al., 1997). Adversely to the previous findings (Benlioglu et al., 2005), no effective weed control was provided by $\mathrm{CM}$ treatment alone.

\section{Conclusions}

In conclusion this study showed that, in the climatic conditions of Southern Italy, greenhouse
SOL alone may provide an effective suppression of root-knot nematodes and weeds, with no strict need for combination with other control tools, as chemicals or organic amendments. Moreover, SOL confirmed its positive long-term effects in vegetable crop systems. In our study, marketable yield increases were clearly evident up to two years from the heating soil treatment.

However, the integration of solar heating with other techniques could be needed in less favourable climates and in field conditions to enhance heat effects and to shorten the solarization period without reducing its efficacy.

\section{Acknowledgements}

This work was carried out within the research project "Innovative and eco-compatible technologies for extra-seasonal and quality vegetable production" (TEPORE), POM Measure.

\section{References}

Bell C.E., Elmore C.L. 1983. Soil solarization as a weed control method in fall planted cantaloupes. Proc. West. Soc. Weed Sci., 36:174-177.

Benlioğlu S., Boz Ö., Yildiz A., Kaşkavalci G., Benlioğlu K. 2005. Alternative Soil Solarization Treatments for the Control of Soil-borne Diseases and Weeds of Strawberry in the Western Anatolia of Turkey. Journal of Phytopathology, 153:423-430.

Candido V., D'Addabbo T., Basile M., Castronuovo D., Miccolis V. 2008. Greenhouse soil solarization: effect on weeds, nematodes and yield of tomato and melon. Agron. Sustain. Dev., 28:221-230.

Carrera L.M., Buyer J.S., Vinyard B., Abdul-Baki A.A., Sikora L.J., Teasdale J.R. 2007. Effects of cover crops, compost, and manure amendments on soil microbial community structure in tomato production systems. Applied Soil Ecology, 37:247-255.

Cartia G. 1998. Solarization in integrated management systems for greenhouses. In: Stapleton J.J., DeVay J.E., Elmore C.L. (eds.): Proceedings of the Second International Conference on Soil Solarization and Integrated Management of Soil-borne pests, 16-21 March 1997, Aleppo, Syrian Arab Republic. FAO Plant Protection and Production Paper 147, Rome, Italy, 333-350.

Clark G.J., Dodgshun N., Sale P.W.G., Tang C. 2007. Changes in chemical and biological properties of a sodic clay subsoil with addition of organic amendments. Soil Biology and Biochemistry, 39:2806-2817.

Coolen W.A. 1979. Methods for the extraction of Meloidogyne spp. and other nematodes from roots and soil. In: Lamberti F., Taylor C.E. (eds.): Root-knot 
nematodes (Meloidogyne spp.). Systematics, Biology and Control, 317-329. Academic Press, London, UK.

Chase C.A., Sinclair T.R., Shilling D.G., Gilreath J.P., Locascio S.J. 1998. Light effects on rhizome morphogenesis in nutsedges (Cyperus spp.): implications for control by soil solarization. Weed Sci., 46:575-580.

D'Addabbo T., Sasanelli N., Lamberti F., Greco P. and Carella A. 2003. Olive pomace and chicken manure amendments for control of Meloidogyne incognita over two crop cycles. Nematropica, 33:1-7.

Dahlquist R.M., Prather T.S., Stapleton J.J. 2007. Time and Temperature Requirements for Weed Seed Thermal Death. Weed Sci., 55:619-625.

Egley G.H. 1990. High-temperature effects on germination and survival of weed seeds in soil. Weed Sci., 38:429-435.

Eshel D., Gamliel A., Grinstein A., Di Primo P., Katan J. 2000. Combined soil treatments and sequence of application in improving the control of soilborne pathogens. Phytopathology, 90:751-757.

Fritsch H.J., Huber R. 1995. Basamid granular - a halogen free soil disinfestant. Acta Hort., 382:76-85.

Gamliel A., Austerweil M., Kritzman G., Perez I. 1999. Combined organic amendments with soil heating to control soilborne plant pathogens. Proceeding of 1999 Annual International Research Conference on Methyl Bromide Alternatives and Emissions Reductions, San Diego, California, USA, 27:1-2.

Gamliel A., Stapleton J.J. 1993. Effect of soil amendment with chicken compost or ammonium phosphate and solarization on pathogen control, rhizosphere microorganisms, and lettuce growth. Plant Dis., 77:886-891.

Giannakou I.O, Sidiropoulos A., Prophetou-Athanasiadou D. 2002. Chemical alternatives to methyl bromide for the control of root-knot nematodes in greenhouses. Pest Management Science, 58:290-296.

Gilreath J.P., Motis T.N., Santos B.M., Noling J.W., Locascio S.J., Chellemi D.O. 2005. Resurgence of soilborne pests in double-cropped cucumber after application of methyl bromide chemical alternatives and solarization in tomato. Hort. Technology; 15:797-801.

Gilreath J.P., Jones J.P., B.M. Santos, Overman A.J. 2004. Soil fumigant evaluations for soilborne pest and Cyperus rotundus control in fresh market tomato. Crop Protection, 23:889-893.

Haidar M.A., Sidahmed M.M. 2000. Soil solarization and chicken manure for the control of Orobanche crenata and other weeds in Lebanon. Crop Protection, 19:169-173.

Hoitink H.A.J., Boehm M.J. 1999. Biocontrol within the context of soil microbial communities: a substrate-dependent phenomenon. Annu. Rev. Phytopathol, 37:427-446.

Ioannou N. 2000. Soil solarization as a substitute for methyl bromide fumigation in greenhouse tomato production in Cyprus. Phytoparasitica, 28:1-9.

Kafikavalci G. 2007. Effects of Soil Solarization and Or- ganic Amendment Treatments for Controlling Meloidogyne incognita in Tomato Cultivars in Western Anatolia. Turk J. Agric. For., 31:159-167.

Kaplan M., Noe J.P., Hartel P.G. 1992. The role of microbes associated with chicken litter in the suppression of Meloidogyne arenaria. Journal of Nematology, 24:522-527.

Katan J., DeVay J.E. (eds.) 1991. Solarization. CRC Press Boca Raton, FL, USA, 267 pp.

Koenning S.R., Barker K.R. 2004. Influence of Poultry Litter Applications on Nematode Communities in Cotton Agroecosystems. Journal of Nematology, 36, 4:524-533.

Landschoot P.J., Park B.S., McNitt A.S., Fidanza M.A. 2004. Effect of dazomet on annual bluegrass emergence and creeping bentgrass establishment in putting green turf. HortScience, 39:1478-1482.

Locascio S.J., Gilreath J.P., Dickson D.W., Kucharek T.A., Jones J.P., Noling J.W. 1997. Fumigant alternatives to methyl bromide for polyethylene-mulched tomato. HortScience, 32:1208-1211.

López Escudero F.J., Blanco López M.A. 2001. Effect of a single or double soil solarization to control Verticillium wilt in established olive orchards in Spain. Plant Dis., 85:489-496.

Nagesh M., Parvatha Reddy P. 2005. Management of carnation and gerbera to control the root-knot nematode, Meloidogyne incognita, in commercial polyhouses. Nematologia Mediterranea, 33:157-162.

Nico A.J., Jimenez-Diaz R.M., Castillo P. 2004. Control of root-knot nematodes by composted agro-industrial wastes in potting mixtures. Crop Protection, 23:581-587.

Noling J.W., Becker J.O. 1994. The challenge of research and extension to define and implement alternatives to methyl bromide. Journal of Nematology, 26, 4S:573-586.

Oka Y., Shapira N., Fine P. 2007. Control of root-knot nematodes in organic farming systems by organic amendments and soil solarization. Crop Protection, 26:1556-1565.

Oka Y., Yermiyahu U. 2002. Nematode-suppressive effects of composts against the root-knot nematode Meloidogyne javanica on tomato. Nematology, 4:891-898.

Opperman M.H., Wood M., Harris P.J., Cherrett C.P. 1993. Nematode and nitrate dynamics in soils treated with cattle slurry. Soil Biology and Biochemistry, 25:19-24.

Patricio F.R.A., Sinigaglia. C., Barros B.C., Freitas S.S., Tessarioli Neto J., Cantarella H., Ghini R. 2006. Solarization and fungicides for the control of drop, bottom rot and weeds in lettuce. Crop Prot., 25:31-38.

Peachey R.E., Pinkerton J.N., Ivors K.L., Miller M.L., Moore L.W. 2001. Effect of soil solarization, cover crops, and metham on field emergence and survival of buried annual bluegrass (Poa annua) seeds. Weed Technol, 15:81-88.

Reddy K.C., Reddy S.S., Malik R.K., Lemunyon J.L., 
Reeves D.W. 2008. Effect of Five-Year Continuous Poultry Litter Use in Cotton Production on Major Soil Nutrients. Agron. J., 100:1047-1055.

Restuccia G., Marchese M., Mauromicale G. 1994. Solarizzazione e lotta contro le infestanti. Riv. Agron., 28:21-30

Riegel C., Fernandez F.A., Noe J.P. 1996. Meloidogyne incognita infested soil amended with chicken litter. J. Nematol., 28:69-378.

Rubin B., Benjamin J. 1984. Solar heating of the soil: involvement of environmental factors in the weed control process. Weed Sci., 32:138-142.

Ruzo L.O. 2006. Physical, chemical and environmental properties of selected chemical alternatives for the pre-plant use of methyl bromide as soil fumigant. Pest Management Science, 62:99-113.

Scopa A., Dumontet S. 2007. Soil Solarization: Effects on Soil Microbiological Parameters. Journal of Plant Nutrition, 30:537-547.

Singla S.L., Pareek A., Grover A. 1997. High temperature. In: Prasad M.N.V. (ed.): Plant Ecophysiology, 101-127. J. Wiley, New York, USA.

Stapleton J.J. 2000. Soil solarization in various agricultural production systems. Crop Prot., 19:837-841.

Stapleton J.J., Heald C.M. 1991. Management of phy- toparasitic nematodes by soil solarization. In: Katan J., DeVay J.E. (eds.): Soil Solarization, 51-60. CRC Press, Boca Raton, FL., USA.

Stapleton J.J., Molinar R.H., Lynn-Patterson K., Mc Feeters S.K., Shrestha A. 2005. Soil solarization provides weed control for limited-resource and organic growers in warmer climates. Calif. Agric., 59:84-89.

Stevens C., Khan V.A., Rodriguez-Kabana R., Ploper L.D, Backman P.A., Collins D.J., Brown J.E., Wilson M.A., Igwegbe E.C.K. 2003. Integration of soil solarization with chemical, biological and cultural control for the management of soilborne diseases of vegetables. Plant Soil, 253:493-506.

Taylor A.L., Sasser J.N. 1987. Biology, Identification and Control of Root-knot Nematodes (Meloidogyne species). North Caroline State University Graphics, Raleigh, North Caroline, USA.

Trudgill D.L., Blok V.C. 2001. Apomictic, polyphagous root-knot nematodes: exceptionally successful and damaging biotrophic root pathogens. Annu. Rev. Phytopathol., 39:53-77.

Yucel S., Ozarslandan A., Colak A., Ay T., Can C. 2007. Effect of Solarization and Fumigant Applications on Soilborne Pathogens and Root-knot Nematodes in Greenhouse-Grown Tomato in Turkey. Phytoparasitica, 35, 5:450-456. 